\title{
Rice Quality Evaluating and Key Quality Genes Genotyping of Rice Germplasm Resources from Africa and Brazil
}

Xu Huashan ${ }^{1}$, He Wei ${ }^{1,2}$, Zhou Lei ${ }^{1}$, Liu Kai ${ }^{1}$, Yang Xiaolin ${ }^{3}$, You Aiqing ${ }^{1,4} \square$

1 Hubei Key Laboratory of Food Crop Germplasm and Genetic Improvement, Institute of Food Crops, Hubei Academy of Agricultural Sciences, Wuhan, 430064, P.R. China

2 Graduate School of Wuhan University, Wuhan, 430072, P.R. China

3 Institute of Plant Protection Soil and Fertilizer, Hubei Academy of Agricultural Sciences, Wuhan, 430064, P.R. China

4 Hubei Collaborative Innovation Center for Grain Industry, Yangtze University, Jingzhou, 434025, P.R. China

\ Corresponding author email: aq you@163.com

Rice Genomics and Genetics, 2021, Vol.12, No.5 doi: 10.5376/rgg.2021.12.0005

Received: 03 Sep., 2021

Accepted: 15 Sep., 2021

Published: 24 Sep., 2021

Copyright (c) 2021 Xu et al., This article was first published in Molecular Plant Breeding in Chinese, and here was authorized to translate and publish the paper in English under the terms of Creative Commons Attribution License, which permits unrestricted use, distribution, and reproduction in any medium, provided the original work is properly cited.

Preferred citation for this article:

Xu H.S., He W., Zhou L., Liu K., Yang X.L., and You A.Q., 2021, Rice quality evaluate and key quality genes genotyping of rice germplasm from Africa and Brazil, Rice Genomics and Genetics, 12(5): 1-8 (doi: 10.5376/rgg.2021.12.0005)

\begin{abstract}
High quality is one of the most objectives in rice breeding, and introducing and evaluating the quality of rice germplasm are very important for screening novel parent with high quality and breeding high quality varieties. In this study, a total of 28 rice germplasm from Africa and Brazil were evaluated their milling quality, appearance quality and cooking and eating quality in Wuhan City, Hubei Province. The results showed the milling quality and appearance quality of most varieties were very high, but the cooking and eating quality of them were bad. Finally, 13 varieties with high quality were screened. The correlation analysis indicated that different traits among milling quality, appearance quality and cooking and eating quality had low correlation between each other. Further, all germplasm was genotyping three important quality genes, GS3,Wx and $A L K$. There were two genotypes in each of GS3 and $W x$, and $A L K$ had three genotypes. The phenotypes showed significantly difference between different genotypes for both $W x$ and $A L K$. Our results will give valuable germplasm resources, gene resources and marker resources for breeding high quality rice varieties.
\end{abstract}

Keywords Germplasm; Quality; Genotype

Rice is the most important grain crop in China. In recent years, with the improvement of people's living standards, people pay more and more attention to the quality of rice (Qiu et al., 2017a; 2017b; Qiu et al., 2020). Since the ' $13^{\text {th }}$ Five-Year Plan', China has attached great importance to the improvement of rice quality (Xu et al., 2020). In 2018 and 2019, the national evaluating of high quality rice varieties was held and a batch of high quality rice varieties with excellent eating quality were identified. However, because breeders have always regarded increasing yield as the primary breeding goal and ignored the improvement of rice quality, the quality of Chinese rice varieties, especially hybrid rice varieties, is poor, and there has been a great gap between Chinese and foreign high quality rice (Ou et al., 2016). Therefore, breeding high quality rice varieties is of great significance to meet consumers' need (Zhang et al., 2010).

Rice quality includes milling quality, appearance quality, cooking and eating quality and nutritional quality. The milling quality includes brown rice rate, milled rice rate and head milled rice rate. The appearance quality mainly includes grain shape and chalkiness. The cooking and eating quality mainly includes amylose content, gel consistence and gelatinization temperature. The nutritional quality mainly includes protein and fat content (Qiu et al., 2015). Among them, head milled rice rate and chalkiness are the core indexes of rice quality, and amylose content is the restrictive index of rice quality classification (Shen et al., 2019). At present, researchers have cloned several genes affecting rice quality, among which GS3 has the greatest impact on grain shape (Fan et al., 2009), amylose content is mainly controlled by $W x$ gene (Wang et al., 1990), and gelatinization temperature is mainly controlled by $A L K$ gene (Gao et al., 2003). Each gene is divided into multiple alleles. Clarifying the phenotypic values corresponding to different alleles and their distribution in germplasm resources can help breeders screen suitable parents and improve the efficiency of breeding high quality rice. 
The screening of high quality germplasm resources is the key to breeding high quality rice varieties. Therefore, it is of great significance to introduce, evaluate and screen high quality rice germplasm resources (Chen et al., 2009). For example, the high quality rice variety 'Meixiangzhan 2' has the genetic relationship with the American high quality rice variety 'Lemont' (Zhou et al., 2005), and the Wuchang rice variety 'Daohuaxiang 2' has some genetic relationship with the Japanese high quality rice variety 'Yueguang' (http://www.ricedata.cn/index.htm). The research showed that in recent years, the genetic background of Chinese high quality rice varieties has become more and more narrow, and it is urgent to introduce new high quality rice germplasm resources from abroad to expand the genetic background.

This study introduced 28 rice germplasm resources from Africa and Brazil, investigated and identified the quality of these germplasm resources in Wuhan, Hubei Province, and identified the genotypes of important quality genes by molecular markers, so as to provide parent resources and allele resources for breeding high quality rice.

\section{Results and Analysis}

\subsection{Quality performance of 28 rice germplasm resources}

The brown rice rate and milled rice rate of 28 germplasm resources showed little diversity among different introduced germplasms (Table 1), and the coefficients of variation were $3.38 \%$ and $4.07 \%$ respectively, which was significantly lower than the head milled rice rate (10.47\%). Among them, the head milled rice rate of 22 germplasm resources exceeded 56\% (first degree of national standard), indicating that the milling quality of these germplasm resources was excellent as a whole. In terms of grain shape, the grain length of 7 germplasm resources from Africa was shorter than $9.0 \mathrm{~mm}$, and the others were long grains; The grain width of 18 germplasm resources was shorter than $2.8 \mathrm{~mm}$, and the others were narrow grains; Finally, except QG049 and QG046, the length to width ratio of other germplasm resources was higher than 3.0, especially the length to width ratio of five germplasm resources from Brazil was higher than 4.0. In addition, the percentage of grains with chalkiness of 22 germplasm resources was lower than $20 \%$, the degree of endosperm with chalkiness of 23 germplasm resources was lower than 5\% (second degree of national standard), and the clarity of 19 germplasm resources was lower than degree 3. The results of grain shape, chalkiness and clarity showed that the appearance quality of these germplasm resources was excellent. Among the 28 germplasm resources, the amylose content of 15 germplasm resources exceeded the range of $14 \% \sim 24 \%$ (high quality rice standard), which was poor. The gel consistence of all germplasm resources was higher than $60 \mathrm{~mm}$, which was excellent. The alkali spreading value of 24 germplasm resources was lower than 5.0, indicating that the gelatinization temperature of most germplasm resources was low. The results showed that the cooking and eating quality of 28 germplasm resources was bad. Referring to the national standard GB/T17891-2017, 13 germplasm resources were high quality rice, including 8 at the first degree, 4 at the second degree and 1 at the third degree.

\subsection{Correlation between different qualities}

The correlation between different quality traits of 28 germplasm resources was shown in Table 2. Among the three traits of milling quality, there was a significant positive correlation between brown rice rate and milled rice rate. Among the six traits of appearance quality, except that the correlation between grain length and grain width, and the correlation between clarity and the other traits was not significant, there was a very significant positive or negative correlation between the other traits, indicating that the correlation between different traits of appearance quality was strong. There was no significant correlation between 3 traits of cooking and eating quality. The results showed that the correlation between different traits of milling quality and cooking and eating quality was low, while the correlation between different traits of appearance quality was strong.

The correlation between different types of quality traits showed that milled rice rate and percentage of grains with chalkiness had a significant positive correlation with amylose content, and a significant negative correlation with grain length. The correlation between other traits of milling quality and the traits of appearance quality and cooking and eating quality was not significant. There was a significant negative correlation between grain length and amylose content, a significant positive correlation between grain width and alkali spreading value, a significant positive correlation between clarity and gel consistence, and no significant correlation between the other traits of appearance quality and cooking and eating quality. The results showed that the correlation between milling quality, appearance quality and cooking and eating quality was low. 
Rice Genomics and Genetics 2021, Vol.12, No.5, 1-8

http://cropscipublisher.com/index.php/rgg

Table 1 Quality performance of 28 rice germplasm resources

\begin{tabular}{|c|c|c|c|c|c|c|c|c|c|c|c|c|c|}
\hline Variety & $\begin{array}{l}\text { Brown rice } \\
\text { rate }(\%)\end{array}$ & $\begin{array}{l}\text { Milled } \\
\text { rice rate } \\
(\%)\end{array}$ & $\begin{array}{l}\text { Head } \\
\text { milled rice } \\
\text { rate }(\%)\end{array}$ & $\begin{array}{l}\text { Grain } \\
\text { length } \\
(\mathrm{mm})\end{array}$ & $\begin{array}{l}\text { Grain width } \\
(\mathrm{mm})\end{array}$ & $\begin{array}{l}\text { Length to } \\
\text { width } \\
\text { ratio }\end{array}$ & $\begin{array}{l}\text { Percentage of } \\
\text { grains with } \\
\text { chalkiness }(\%)\end{array}$ & $\begin{array}{l}\text { Degree of } \\
\text { endosperm with } \\
\text { chalkiness }(\%)\end{array}$ & Clarity & $\begin{array}{l}\text { Amylose } \\
\text { content } \\
(\%)\end{array}$ & $\begin{array}{l}\text { Gel } \\
\text { consistence } \\
(\mathrm{mm})\end{array}$ & $\begin{array}{l}\text { Alkali } \\
\text { spreading } \\
\text { value }\end{array}$ & Degree $^{a}$ \\
\hline QG006 & 67.93 & 58.02 & 49.55 & 9.10 & 2.35 & 3.82 & 8.18 & 1.89 & 2 & 26.62 & 120.00 & 2.20 & Substandar \\
\hline QG017 & 79.87 & 67.76 & 51.50 & 9.25 & 2.53 & 3.66 & 9.35 & 1.81 & 2 & 20.78 & 114.70 & 5.80 & 2 \\
\hline QG018 & 78.08 & 66.53 & 56.03 & 9.31 & 2.58 & 3.61 & 3.15 & 0.86 & 1 & 19.38 & 130.70 & 5.20 & 1 \\
\hline QG020 & 79.86 & 68.80 & 61.02 & 8.75 & 2.39 & 3.63 & 5.87 & 1.05 & 2 & 27.30 & 125.70 & 2.00 & Substandar \\
\hline QG039 & 80.19 & 68.79 & 58.47 & 9.59 & 2.62 & 3.47 & 20.32 & 2.26 & 3 & 27.13 & 138.70 & 2.30 & Substandar \\
\hline QG040 & 79.14 & 69.27 & 57.70 & 9.47 & 2.59 & 3.66 & 7.76 & 1.48 & 2 & 20.06 & 95.30 & 2.20 & 1 \\
\hline QG041 & 77.77 & 68.31 & 46.55 & 9.17 & 2.91 & 3.16 & 22.36 & 5.71 & 2 & 27.62 & 65.00 & 5.80 & Substandar \\
\hline QG044 & 79.30 & 71.47 & 67.02 & 8.10 & 2.38 & 3.41 & 11.52 & 1.89 & 3 & 28.32 & 133.00 & 2.20 & Substandar \\
\hline QG046 & 78.44 & 67.51 & 57.03 & 7.81 & 2.94 & 2.68 & 81.96 & 21.05 & 2 & 28.18 & 92.50 & 2.20 & Substandar \\
\hline QG049 & 80.13 & 72.43 & 63.39 & 8.12 & 3.18 & 2.56 & 81.40 & 15.56 & 4 & 26.63 & 130.50 & 2.30 & Substandar \\
\hline QG052 & 78.41 & 66.09 & 58.28 & 9.14 & 2.85 & 3.22 & 13.96 & 5.43 & 3 & 17.39 & 122.00 & 4.30 & 3 \\
\hline QG053 & 77.82 & 67.10 & 59.22 & 9.54 & 2.70 & 3.55 & 30.00 & 4.12 & 2 & 17.20 & 124.70 & 4.50 & 2 \\
\hline QG061 & 77.69 & 66.11 & 61.08 & 9.19 & 2.75 & 3.34 & 6.06 & 0.50 & 3 & 13.18 & 114.00 & 2.70 & Substandar \\
\hline QG064 & 79.16 & 70.14 & 63.15 & 8.64 & 2.92 & 3.00 & 35.29 & 5.13 & 2 & 27.02 & 66.70 & 4.20 & Substandar \\
\hline QG068 & 81.25 & 69.20 & 56.92 & 9.29 & 2.82 & 3.30 & 7.69 & 3.58 & 3 & 23.78 & 81.00 & 2.30 & 2 \\
\hline Sahel 108 & 79.79 & 68.07 & 46.49 & 8.67 & 2.38 & 3.66 & 10.00 & 2.41 & 2 & 25.91 & 134.50 & 2.20 & Substandar \\
\hline Sahel 134 & 79.29 & 69.10 & 42.52 & 8.95 & 2.30 & 3.90 & 19.23 & 2.07 & 4 & 29.38 & 142.00 & 2.30 & Substandar \\
\hline$Z Y-1$ & 81.22 & 67.98 & 62.53 & 9.71 & 2.19 & 4.46 & 5.43 & 1.70 & 2 & 23.20 & 68.00 & 2.00 & 1 \\
\hline $\mathrm{ZY}-2$ & 79.66 & 67.61 & 63.54 & 9.14 & 2.32 & 3.97 & 5.46 & 1.17 & 2 & 21.53 & 88.50 & 2.50 & 1 \\
\hline $\mathrm{ZY}-3$ & 79.72 & 66.90 & 48.42 & 10.56 & 2.47 & 4.30 & 5.35 & 1.26 & 2 & 26.68 & 81.30 & 6.00 & Substandar \\
\hline ZY-4 & 80.67 & 63.53 & 59.90 & 9.89 & 2.55 & 3.89 & 3.63 & 0.56 & 2 & 13.16 & 73.70 & 2.00 & Substandar \\
\hline$Z Y-5$ & 79.75 & 66.03 & 59.83 & 9.70 & 3.10 & 3.14 & 18.47 & 3.20 & 3 & 16.88 & 102.30 & 4.50 & 2 \\
\hline ZY-7 & 84.91 & 68.11 & 62.32 & 9.15 & 2.23 & 4.13 & 7.30 & 1.67 & 2 & 22.90 & 109.30 & 2.00 & 1 \\
\hline ZY-8 & 77.28 & 62.99 & 59.24 & 9.41 & 2.99 & 3.16 & 6.09 & 0.94 & 2 & 15.91 & 81.30 & 4.30 & 1 \\
\hline ZY-9 & 78.56 & 67.22 & 57.31 & 9.20 & 2.29 & 4.05 & 5.02 & 1.17 & 2 & 22.72 & 71.50 & 2.00 & 1 \\
\hline ZY-16 & 78.03 & 64.82 & 60.13 & 9.47 & 3.02 & 3.13 & 6.25 & 0.84 & 2 & 15.49 & 64.30 & 4.50 & 1 \\
\hline ZY-17 & 79.16 & 67.50 & 63.06 & 9.52 & 2.33 & 4.11 & 7.38 & 1.33 & 2 & 26.00 & 108.50 & 2.00 & Substandar \\
\hline ZY-25 & 76.76 & 65.81 & 60.52 & 9.31 & 2.91 & 3.21 & 5.38 & 1.20 & 3 & 25.48 & 104.70 & 2.00 & Substandar \\
\hline
\end{tabular}

Note: a: The classification of quality grade shall refer to the national standard GB/T17891-2017 
Rice Genomics and Genetics 2021, Vol.12, No.5, 1-8

http://cropscipublisher.com/index.php/rgg

Table 2 Correlation coefficients of different quality traits in the 28 rice germplasm resources

\begin{tabular}{|c|c|c|c|c|c|c|c|c|c|c|c|}
\hline Index of quality & $\begin{array}{l}\text { Brown rice } \\
\text { rate }(\%)\end{array}$ & $\begin{array}{l}\text { Milled rice } \\
\text { rate }(\%)\end{array}$ & $\begin{array}{l}\text { Head milled } \\
\text { rice rate }(\%)\end{array}$ & $\begin{array}{l}\text { Grain } \\
\text { length } \\
(\mathrm{mm})\end{array}$ & $\begin{array}{l}\text { Grain width } \\
(\mathrm{mm})\end{array}$ & $\begin{array}{l}\text { Length to } \\
\text { width ratio }\end{array}$ & $\begin{array}{l}\text { Percentage of } \\
\text { grains with } \\
\text { chalkiness }(\%)\end{array}$ & $\begin{array}{l}\text { Degree of } \\
\text { endosperm with } \\
\text { chalkiness (\%) }\end{array}$ & Clarity & $\begin{array}{l}\text { Amylose } \\
\text { content }\end{array}$ & $\begin{array}{l}\text { Gel } \\
\text { consistence }\end{array}$ \\
\hline Brown rice rate & $0.68 * *$ & - & - & - & - & - & - & - & - & - & - \\
\hline Head milled rice rate & 0.28 & 0.21 & - & - & - & - & - & - & - & - & - \\
\hline Grain length & 0.05 & $-0.40^{*}$ & -0.15 & - & - & - & - & - & - & - & - \\
\hline Grain width & -0.11 & 0.01 & 0.14 & -0.15 & - & - & - & - & - & - & - \\
\hline Length to width ratio & 0.15 & -0.17 & -0.16 & $0.56^{* *}$ & $-0.89 * *$ & - & - & - & - & - & - \\
\hline $\begin{array}{l}\text { Percentage of grains } \\
\text { with chalkiness }\end{array}$ & 0.03 & $0.37^{*}$ & 0.07 & $-0.63 * *$ & $0.50 * *$ & $-0.65 * *$ & - & - & - & - & - \\
\hline $\begin{array}{l}\text { Degree of endosperm } \\
\text { with chalkiness }\end{array}$ & 0.02 & 0.30 & 0.03 & $-0.63 * *$ & $0.48 * *$ & $-0.63 * *$ & $0.96^{* *}$ & - & - & - & - \\
\hline Clarity & 0.10 & 0.35 & 0.02 & -0.26 & 0.28 & -0.35 & 0.33 & 0.24 & - & - & - \\
\hline Amylose content & -0.03 & $0.43 *$ & -0.27 & $-0.44 *$ & -0.26 & 0.03 & 0.33 & 0.32 & 0.20 & -- & - \\
\hline Gel consistence & -0.08 & 0.17 & -0.12 & -0.33 & -0.19 & -0.05 & 0.12 & 0.04 & $0.39 *$ & 0.23 & - \\
\hline Alkali spreading value & -0.09 & -0.11 & -0.32 & 0.35 & $0.38^{*}$ & -0.16 & -0.05 & -0.06 & -0.29 & -0.25 & -0.20 \\
\hline
\end{tabular}

Note: $*$ and $* *$ represent $\mathrm{p}<0.05$ and 0.01 respectively 


\subsection{Genotypes of the important genes in 28 rice germplasm resources}

$G S 3, W x$ and $A L K$ are the most important quality genes cloned at present. We identified the genotypes of these three genes in 28 germplasm resources by molecular marker or sequencing (Table 3). According to the C/A mutation in the second exon, GS3 was divided into two genotypes, long-grain genotypes and short-grain genotypes. Except that QG041, QG044, QG052 and QG053 were short-grain genotypes, the others were all long-grain genotypes. However, among these 4 varieties of short-grain genotype, the grain length of 3 varieties was longer than $9.0 \mathrm{~mm}$, while among the varieties of long-grain genotype, the grain length of 6 varieties was shorter than $9.0 \mathrm{~mm}$. $W x$ was divided into two genotypes, $W x^{a}$ and $W x^{b}$, the number of which was 17 and 11 respectively. The amylose content of $W x^{a}$ germplasm was $22.72 \% \sim 29.28 \%$, which was significantly higher than that of $W x^{b}$ germplasm (13.16\% 21.53\%). After sequencing the $A L K$ gene and comparing it with Nipponbare, a reference sequence, we found that there was an $\mathrm{A} / \mathrm{G}$ mutation at 3797 , resulting in the encoded serine being mutated to glycine, where five germplasm resources from Africa had mutations. There was a T/G mutation at 3 901, but the mutation did not change the amino acid coding, and the mutated germplasm resources were the same as 3 797. There was a $\mathrm{A} / \mathrm{G}$ mutation at 4 196, resulting in the encoded methionine being mutated to valine, where all germplasm resources had mutations. There were two GC/TT mutations at 4327 and 4328 , resulting in the encoded glycine and leucine being mutated to glycine and phenylalanine, where 10 germplasm resources had mutations. Finally, $A L K$ was divided into three genotypes by using mutations at 3 797, 3 901, 4327 and 4328 . Among them, the number of germplasm resources with ATGC and GCGC genotypes was 13 and 5 respectively, with alkali spreading value being 2.48 and 2.18 respectively, which were significantly lower than that of ATTT (alkali spreading value was 4.53).

Table 3 Genotypes of three important quality genes in 28 rice germplasm resources

\begin{tabular}{|c|c|c|c|}
\hline \multirow[t]{2}{*}{ Variety } & \multicolumn{3}{|l|}{ Gene } \\
\hline & $\overline{G S 3}$ & $W x$ & $A L K$ \\
\hline QG006 & $\mathrm{L}$ & $W x^{a}$ & ATGC \\
\hline QG017 & $\mathrm{L}$ & $W x^{b}$ & ATTT \\
\hline QG018 & $\mathrm{L}$ & $W x^{b}$ & ATTT \\
\hline QG020 & $\mathrm{L}$ & $W x^{a}$ & GGGC \\
\hline QG039 & $\mathrm{L}$ & $W x^{a}$ & ATGC \\
\hline QG040 & $\mathrm{L}$ & $W x^{b}$ & ATTT \\
\hline QG041 & S & $W x^{a}$ & ATTT \\
\hline QG044 & S & $W x^{a}$ & GGGC \\
\hline QG046 & $\mathrm{L}$ & $W x^{a}$ & GGGC \\
\hline QG049 & $\mathrm{L}$ & $W x^{a}$ & ATGC \\
\hline QG052 & S & $W x^{b}$ & ATTT \\
\hline QG053 & S & $W x^{b}$ & ATTT \\
\hline QG061 & $\mathrm{L}$ & $W x^{b}$ & ATGC \\
\hline QG064 & $\mathrm{L}$ & $W x^{a}$ & ATGC \\
\hline QG068 & $\mathrm{L}$ & $W x^{a}$ & ATGC \\
\hline Sahel 108 & $\mathrm{~L}$ & $W x^{a}$ & GGGC \\
\hline Sahel 134 & $\mathrm{~L}$ & $W x^{a}$ & GGGC \\
\hline ZY-1 & $\mathrm{L}$ & $W x^{a}$ & ATGC \\
\hline$Z Y-2$ & $\mathrm{~L}$ & $W x^{b}$ & ATTT \\
\hline$Z Y-3$ & $\mathrm{~L}$ & $W x^{a}$ & ATTT \\
\hline ZY-4 & $\mathrm{L}$ & $W x^{b}$ & ATGC \\
\hline ZY-5 & $\mathrm{L}$ & $W x^{b}$ & ATTT \\
\hline$Z Y-7$ & $\mathrm{~L}$ & $W x^{a}$ & ATGC \\
\hline ZY-8 & $\mathrm{L}$ & $W x^{b}$ & ATGC \\
\hline ZY-9 & $\mathrm{L}$ & $W x^{a}$ & ATGC \\
\hline$Z Y-16$ & $\mathrm{~L}$ & $W x^{b}$ & ATTT \\
\hline ZY-17 & $\mathrm{L}$ & $W x^{a}$ & ATGC \\
\hline$Z Y-25$ & $\mathrm{~L}$ & $W x^{a}$ & ATGC \\
\hline
\end{tabular}




\section{Discussion}

With the development of China's economy and the improvement of people's living standards, the quality of rice has attracted more and more attention. However, for a long time, China's rice breeding had always taken high yield as the primary breeding goal, resulting in a shortage of high quality rice varieties (Ou et al., 2016). Although some high quality rice varieties are widely popularized in production, there is still an urgent need for breeders to screen and breed more new high quality rice varieties that can meet different production needs. The screening and breeding of high quality rice depends on the exploration and utilization of high quality germplasm resources, so the introduction and identification of high quality germplasm resources is very important for breeding high quality rice. Guangdong Academy of Agricultural Sciences started the breeding of hybrid rice with high quality earlier in China (Zhou et al., 2005). In the 1970s, the Academy began to introduce various high quality germplasm resources as parents. After decades of efforts, it has bred a large number of high quality rice varieties. However, at present, the genetic background of high quality rice germplasm resources in China is very narrow, the screened and bred high quality rice varieties are very close, and the yield is difficult to further improve. Therefore, it is urgent to introduce new high quality germplasm resources from abroad to broaden the current genetic background and raise the breeding of high quality rice to a higher level.

In this study, 28 rice germplasm resources were introduced from Africa and Brazil, and their quality was evaluated in Wuhan City, Hubei Province. The results showed that the milling quality and appearance quality of most varieties in these germplasm resources were very high, but the cooking and eating quality was bad. Finally, 13 germplasm resources reached the standard of high quality rice, including 8 at the first degree, 4 at the second degree and 1 at the third degree. These high quality germplasm resources can be used as important parents for breeding high quality rice. Because rice quality is greatly affected by the environment, it needs to be repeatedly identified in different years and different ecological conditions. In addition, other agronomic characters of these high quality germplasm resources need to be inspected to provide a basis for subsequent high quality rice breeding.

In addition, 15 of the 28 germplasm resources did not meet the standard of high quality rice. It was found that the amylose content of these germplasm resources did not meet the standard, resulting in their being substandard. Among these germplasm resources, the head milled rice rate of 10 germplasm resources exceeded the first degree of national standard (more than 56\%), and the degree of endosperm with chalkiness of 8 germplasm resources reached the first degree of national standard (less than 2\%), indicating that these germplasm resources have great utilization value in some aspect. In the process of screening and breeding high quality rice with these germplasm resources, special attention should be paid to not improving single quality and leading to the decline of other qualities. In addition, the correlation analysis between quality traits showed that the correlation between milling quality, appearance quality and cooking and eating quality was low, indicating that we can only improve one or one kind of quality without causing the decline of other qualities.

We used molecular markers to identify the genotypes of three important quality genes, GS3, Wx and $A L K$, in germplasm resources. There were two genotypes in each of $G S 3$ and $W x$, and $A L K$ had three genotypes. GS3 encoded $\beta$ subunit of $\mathrm{G}$ signal protein (Mao et al., 2010), a C/A mutation in the second exon led to the early termination of the coding protein, resulting in the long-grain phenotype (Fan et al., 2009). The markers used in this study were used to identify the mutation, but most of the germplasm resources of short-grain genotype showed long grains, while 6 varieties of germplasm resources of long-grain genotype showed short grains, indicating that these materials also contained other genes affecting grain shape. In subsequent studies, genetic populations can be constructed to find out new grain shape genes. $W x$ encoded granular starch synthase (Wang et al., 1990), which was the most important gene controlling amylose content in rice. $W x$ was mainly divided into two genotypes. Among them, $W x^{a}$ was a wild type, $W x^{b}$ was evolved from $W x^{a}$, and the amylose content of $W x^{b}$ was significantly lower than that of $W x^{a}$ (Sano et al. 1986), which was consistent with the results of this study. $A L K$ is the only cloned gene controlling gelatinization temperature currently, encoding soluble starch synthase II (Gao et al., 2003). In this study, three genotypes of this gene were found in 28 germplasm resources by 
sequencing, and the alkali spreading value of ATGC and GCGC was significantly lower than that of ATTT. Subsequently, PCR based molecular markers can be developed to identify these two genotypes. In addition, it is difficult to identify the quality phenotype of rice, especially the low-generation single plant. Therefore, using molecular breeding to improve rice quality is the best way to screen and breed high quality rice. The genotype resources of the above three genes are important gene resources for molecular breeding to improve rice quality. These markers (or developing new molecular markers according to the sequencing results) can provide important marker resources for molecular breeding high quality rice, which can greatly accelerate the rate and improve the efficiency of breeding high quality rice.

\section{Materials and Methods}

\subsection{Test materials}

The rice germplasm resources used in this study were from Africa and Brazil, of which 17 germplasm resources with 'QG' numbers and 'Sahel' numbers were from Africa, and 11 germplasm resources with ' $Z Y$ ' numbers were from Brazil.

\subsection{Material planting and quality inspection}

All germplasm resources were planted in the base of Institute of Food Crops, Hubei Academy of Agricultural Sciences in the summer of 2014. All materials were sown uniformly on May $15^{\text {th }}$. The random block design was adopted. Each variety was planted 3 rows, with 10 plants in each row, and the spacing between plants was 20 $\mathrm{cm} \times 25 \mathrm{~cm}$, which was repeated twice. The field management was the same as usual. The heading date of each variety was recorded. $30 \mathrm{~d}$ after heading, mixed harvest was carried out according to the variety. After harvest and threshing, the grain crop was spread out in the sun and dried in the shade in the courtyard of Institute of Food Crops, Hubei Academy of Agricultural Sciences, and then stored at room temperature for 3 months before determining the quality.

The appearance quality of rice varieties, including grain length, grain width, length to width ratio, percentage of grains with chalkiness, degree of endosperm with chalkiness and clarity, was detected by SC-E rice appearance quality detection analyzer of Hangzhou Wseen. According to the national standards GB/T 5495-2008 and GB/T 21719-2008, the milling quality of various rice varieties, including brown rice rate, milled rice rate and head milled rice rate, was determined. According to the industry standard NY147-88 of the Ministry of Agriculture, the amylose content, gel consistence and alkali spreading value were determined.

\subsection{Genotype identification of important quality genes}

DNA extraction was referred to the method of Murry and Thomson (1980). Three markers for three important quality genes were developed by Primer 5.0 software (Table 4). Among them, two were CAPs markers, which were digested with PstI, and one was sequencing marker. The PCR reaction system was based on the system of Fan et al. (2009) and Lv (2018), and PCR products were identified by $1 \%$ agarose gel. The sequencing of $A L K$ gene was completed by Nanjing GenScript Biotechnology Co., Ltd., and the sequence variation of $A L K$ gene in different varieties was compared with Nipponbare, a reference sequence.

Table 4 Information of markers for genotyping target genes (Sequences were provided by Liu Qiaoquan Lab in Yangzhou University)

\begin{tabular}{llll}
\hline Gene & Marker name & Primer sequence & Marker type \\
\hline$G S 3$ & GS3PST-F & TATTTATTGGCTTGATTTCCTGTG & CAPs \\
& GS3PST-R & GCTGGTTTTTTACTTTCATTTGCC & \\
$W x$ & $\mathrm{pAg}$ & GCTTCACTTCTCTGCTTGTG & CAPs \\
& $\mathrm{pBg}$ & ATGATTTAACGAGAGTTGAA & \\
ALK & ALK-F & CAGGGACACGATAAACTCTTCTT & Sequencing \\
& ALK-R & GCATCAATGGACATAACAAACAC & \\
\hline
\end{tabular}

\section{Authors' contributions}

XHS and HW were the designers and executors of the experiments. XHS, HW and ZL completed data analysis and wrote the first draft of the manuscript. LK and YXL participated in designing the experiments and analyzing the results; YAQ was the designer and 
person in charge of the project, guiding experimental design, data analysis, manuscript writing and revision. All authors read and approved the final manuscript.

\section{Acknowledgments}

This study was jointly funded by the National Key Research and Development Program of China (2017YFD0100305), Technical Innovation Project of Hubei Province (2018ABA083), the Youth Fund of the Hubei Academy of Agricultural Sciences (the breeding of new varieties of high quality rice with rice blast resistance gene and fragrance gene), the Open Fund Project of Key Laboratory of Integrated Pest Management on Crops in Central China, Ministry of Agriculture / Hubei Key Laboratory of Crop Diseases, Insect Pests and Weeds Control (2018ZTSJJ12) and the Project of Agricultural Science and Technology Innovation Center in Hubei Province (2018-620-003-001).

\section{References}

Chen Z.G., Deng H.C., and Zhao S.P., 2009, Genetic diversity and relationship of 47 rice varieties revealed by ISSR, Jiyinzuxue Yu Yingyong Shengwuxue (Genomics and Applied Biology), 28(3): 498-502

Fan C.C., Yu S.B., Wang C.R., and Xing Y.Z., 2009, A causal C-A mutation in the second exon of GS3 highly associated with rice grain length and validated as a functional marker, Theor. Appl. Gent., 118: 465-472 https://doi.org/10.1007/s00122-008-0913-1

Gao Z.Y., Zeng D.L., Cui X., Zhou Y.H., Yan M.X., Huang D.N., Li J.Y., and Qian Q., 2003, Map-based cloning of the $A L K$ gene, which controls the gelatinization temperature in rice, Science in China (Series C), 46(6): 661-668 https://doi.org/10.1360/03yc0099

Lv W.K., Hu H., He L.P., Zhang X.C., Ou X.X., Chen H.Y., Yu S.B., Xu J.L., Qiu X.J., Li Z.X., Xu J.Y., Wang X.Y., Liu Z.Y., Wang C.R., Jiang J.F., and Lou J., 2018, Characterization and fine mapping of two white panicle genes with duplicated effect in rice, Int. J. Agric. Biol., 20: 2805-2811

Mao H.L., Sun S.Y., Yao J.L., Wang C.R., Yu S.B., Xu C.G., Li X.H., and Zhang Q.F., 2010, Linking differential domain functions of the GS3 protein to natural variation of grain size in rice, Proc. Natl. Acad. Sci., 107: 19579-19584 https://doi.org/10.1073/pnas.1014419107

Murry M.G., and Thomson W.F., 1980, Rapid isolation of high molecular-weight plant DNA, Nucl. Acid. Res., 8: 4321-4325 https://doi.org/10.1093/nar/8.19.4321

Ou X.X., Hu H., Zhang K., Du B., Liu H., Jiang G.S., Qiu X.J., Xu J.L., Xing D.Y., and Yang L.W., 2016, Screening for high quality Indica rice germplasm resources, Hubei Nongye Kexue (Hubei Agricultural Sciences), 55(1):24-27

Qiu X.J., Chen K., Lv W.K., Ou X.X., Zhu Y.J., Xing D.Y., Yang L.W., Fan F.J., Yang J., Xu J.L., Zheng T.Q., and Li Z.K., 2017a, Examining two sets of introgression lines reveals background-independent and stably expressed QTL that improve grain appearance quality in rice (Oryza sativa L.), Theor. Appl. Genet., 130: 951-967 https://doi.org/10.1007/s00122-017-2862-Z

Qiu X.J., Du B., Hu H., Ou X.X., Lv W.K., Yang L.W., Xing D.Y., Xu J.L., Zheng T.Q., Xu J.Y., Li Z.X., Zhang Y.B., and Wang X.Y., 2017b, Scanning QTLs for grain shape using two sets of introgression lines in rice, Int. J. Agric. Biol., 19: 509-516 https://doi.org/10.17957/IJAB/15.0320

Qiu X.J., Pang Y.L., Yuan Z.H., Xing D.Y., Xu J.L., Michael D., Li Z.K., and Ye G.Y., 2015, Genome-wide association study of grain appearance and milling quality in a worldwide collection of Indica rice germplasm, PLoS One, 10(12): e0145577 https://doi.org/10.1371/journal.pone.0145577

Qiu X.J., Zhu S.B., Hu H., Wang C.C., Lv W.K., He L.P., Xu J.Y., Li Z.X., Xing D.Y., Yang L.W., Wei W.L., and Xu J.L., 2020, Genome-wide association mapping for grain shape in rice accessions, Int. J. Agric. Biol., 23: 612-618

Sano Y., Katsumata M., and Okuno K., 1986, Genetic studies in cultivated rice. 5. Inter- and intraspecific differentiation in the waxy gene expression of rice, Euphytica, 35(1): 1-9 https://doi.org/10.1007/BF00028534

Shen S., Zhao F.W., Qiu X.J., Zhang Y.B., and Tian X.H., 2019, Approaches to improve rice quality and matching cultivation techniques in Hubei province, Zhongguo Daomi (China Rice), 25(3): 140-142

Wang Z.Y., Wu Z.L., Xing Y.Y., Zheng F.G., Guo X.L., Zhang W.G., and Hong M.M., 1990, Nucleotide sequence of rice waxy gene, Nucl. Acid. Res., 18(19): 5898 https://doi.org/10.1093/nar/18.19.5898

Xu C.C., Ji L., Chen Z.D., Zhou X.Y., and Fang F.P., 2020, Analysis of China's rice industry in 2019 and the outlook for 2020, Zhongguo Daomi (China Rice), 26(2): $1-4$

Zhang X.W., Wang J., Tang Y.Q., and Li J.Y., 2010, Aroma genetic breeding and its cultivation of aromatic rice, Jiyinzuxue Yu Yingyong Shengwuxue (Genomics and Applied Biology), 29(3): 550-555

Zhou S.C., Li H., Huang D.Q., and Lu D.C., 2005, Rice core germplasm breeding, Keji Daobao (Science and Technology Review), 23(11): 23-26 